\title{
MÁS ALLÁ DEL CAMBIO CLIMÁTICO: IMPLICACIONES DE LA CONTAMINACIÓN MARINA POR AGROQUÍMICOS SOBRE LAS INTERACCIONES SIMBIÓTICAS Y EL ÉXITO ECOLÓGICO DE LOS CORALES
}

\author{
Beyond Climate Change: The Impact of Agrochemical Pollution on the Symbiotic Interactions and \\ Ecological Success of Corals
}

Héctor Ocampo-Alvarez ${ }^{1}$, Fabián Alejandro Rodríguez-Zaragoza ${ }^{1}$, Amayaly Becerril-Espinosa ${ }^{2 *}$

${ }^{1}$ Laboratorio de Ecología Molecular, Microbiología y Taxonomía, Laboratorio de Ecosistemas Marinos y Acuicultura, Departamento de Ecología, Centro Universitario de Ciencias Biológicas y Agropecuarias, Universidad de Guadalajara, Zapopan, Jalisco, CP 45110, México. ${ }^{2}$ CONACYT, Departamento de Ecología, Centro Universitario de Ciencias Biológicas y Agropecuarias, Universidad de Guadalajara, Zapopan, Jalisco, CP 45110, México

*Autor para correspondencia: amayaly9@gmail.com, abecerril@conacyt.mx

\section{Resumen}

Los arrecifes de coral son ecosistemas marinos altamente productivos que albergan una gran biodiversidad. Los organismos formadores de estos arrecifes son los corales escleractinos, quienes forman interacciones simbióticas con múltiples microorganismos. Una de las interacciones simbióticas más conocidas en estos sistemas es la que establecen con las microalgas Symbiodinium. Las microalgas producen por la vía de la fotosíntesis hasta el 90\% de la energía que requiere el coral. En contraparte, Symbiodinium, recibe del coral un nicho apropiado, protegido del ambiente externo, de la competencia con otros organismos y de la depredación; además proporciona abundantes nutrientes producidos por otros simbiontes del coral de los que destacan las bacterias. Así como esta, en los corales existen múltiples interacciones simbióticas que confieren a los corales las capacidades metabólicas que han habilitado su capacidad de adaptación a cambios climáticos durante millones de años. Sin embargo, en las últimas décadas los ecosistemas de arrecifes de coral están siendo ampliamente diezmados. Ante las nuevas características de un medio ambiente con importantes cambios, a veces un tanto erráticos, es posible que las interacciones que originalmente le brindaban ventajas ecológicas, ya no sean suficientes para sobrepasar las adversidades ambientales o bien que en consecuencia de los cambios generados en el medio ambiente, la diversidad de microorganismos capaces de interaccionar haya sido disminuida a tal grado que las interacciones que se pueden formar con los pocos microorganismos restantes no le confieren a los corales las ventajas adaptativas suficientes para afrontar el reto del cambio climático. En este ensayo argumentamos sobre la posibilidad de que una disminución del stock de microorganismos capaces de interaccionar con los corales, a consecuencia de la contaminación marina, sea una causa de la perdida de aptitud biológica de los corales para sobrevivir en el actual escenario del cambio climático global.

Palabras clave: microbioma de coral, simbiosis, Symbiodinium, cambio climático global, adaptación. 


\section{Abstract}

Coral reefs are highly productive marine ecosystems that harbor a high biodiversity. The forming organisms of these reefs are the scleractinian corals, which form symbiotic interactions with multiple microorganisms. One of the best known symbiotic interactions in these systems is the one established with the microalgae Symbiodinium. The microalgae produce through photosynthesis up to $90 \%$ of the energy required by the coral. On the other hand, Symbiodinium receives from the coral an appropriate niche, that protects Symbiodinium from the external environment, from the competition with other organisms and predation; it also provides abundant nutrients produced by other coral symbionts highlighting the bacteria. As well as this, multiple symbiotic interactions confer metabolic capabilities to corals, which have enabled their capacity to adapt to climate changes for millions of years. However, in recent decades coral reef ecosystems are being extensively decimated. Given the new characteristics of an environment with significant changes sometimes somewhat erratic, probably the interactions that initially provided ecological advantages to corals are no longer sufficient to overcome environmental adversities or that as a result of the changes generated in the environment. The diversity of microorganisms capable of interactions that can be formed with the few remaining microorganisms do not confer to the coral, sufficient adaptative advantages to face the challenge of climate change. In this essay, we argue about the possibility that a decrease in the stock of microorganisms capable of interacting with corals, as a result of marine pollution, is a cause of the loss of biological aptitude of corals to survive in the current global climate change.

Key words: coral microbiome, symbiosis, Symbiodinium, global climate change, adaptation
Durante los últimos años, los arrecifes de coral han sufrido episodios de muertes recurrentes que han causado la pérdida de grandes extensiones de estos ecosistemas. El fenómeno es muy evidente, debido a que los corales se decoloran o blanquean antes de morir (Hughes et al., 2009). La comprensión de los factores que contribuyen al blanqueamiento y muerte de los corales, es sin duda un tema de gran interés científico y de relevancia ambiental, pues con base en el conocimiento de causa, se podrían proponer y establecer estrategias adecuadas para su protección y conservación.

Los arrecifes de coral son sistemas ecológicos de gran belleza escénica, con múltiples formas y matices, donde viven diversos organismos coloridos, que pueden ser observados con facilidad entre los corales durante un buceo recreativo. Este ecosistema es uno de los recursos naturales marinos más carismático ya que atrae a muchos visitantes de todo el mundo. Económicamente, se estima que a nivel mundial generan ingresos anuales por alrededor de 35,000 millones USD (Spalding et al., 2017).

Estos ecosistemas marinos son muy importantes, porque se consideran uno de los más productivos y de mayor biodiversidad en el planeta (Putnam et al., 2017). Sus tasas de producción primaria y de biodiversidad son comparables a los de los bosques lluviosos terrestres (Silveira et al. 2017). Resulta asombroso que a pesar de solo ocupar cerca del $0.1 \%$ de la superficie marina, albergan alrededor del $25 \%$ de toda la biodiversidad marina. La complejidad estructural que generan los corales son un hábitat adecuado que alberga áreas que funcionan como guarderías y sitios de crianza (viveros) de una gran diversidad de organismos en etapas tempranas de desarrollo, como bivalvos, cangrejos, erizos de mar, esponjas, estrellas de mar, gusanos, peces, pulpos, entre otros (Stella et al., 2011). Por ejemplo, el arrecife de coral más grande de 
México, "Banco Chinchorro" que está localizado en el Caribe mexicano, tiene un registro de hasta1,030 especies (Hernández et al., 2008).

Además de albergar una gran diversidad de macroorganismos visibles a simple vista, los arrecifes de coral también hospedan una comunidad importante de microorganismos. Estos son el componente biológico más diverso y abundante en los corales. La suma de todos estos microorganismos se conoce como microbioma y comprende desde hongos microscópicos, microalgas y pequeños protistas, hasta bacterias y virus (Bourne et al., 2016). Al igual que en animales y plantas, los corales interactúan con su microbioma, establecen interacciones simbióticas y obtienen beneficios importantes para su crecimiento, desarrollo y supervivencia ante diversos factores de estrés ambiental.

La interacción mejor conocida de los corales y su microbioma es la que se establece con la microalga Symbiodinium, que vive como endosimbionte, dentro de las células de tejidos internos del coral. En esta interacción simbiótica, la microalga Symbiodinium produce alimento para el coral mediante el proceso de fotosíntesis y favorece la formación del exoesqueleto de carbonato del coral. Por otro lado, Symbiodinium recibe del coral un nicho apropiado para su desarrollo y supervivencia, aislado de las adversidades del ambiente externo y recibiendo protección de la competencia y depredación por parte de otros organismos (Bourne et al., 2016; Davy et al., 2012). La importancia de esta relación es tal, que bajo ciertas condiciones, como el estrés térmico, se produce la pérdida de la microalga simbionte, generando el blanqueamiento del coral y eventualmente su muerte por inanición (Boulotte et al., 2016; Fabina et al., 2013).

Anteriormente, se consideraba que la relación entre el coral y el simbionte fotosintético era especie-específica y que sólo se podía heredar de las células progenitoras a las células hijas. Sin embargo, evidencias recientes indican que en un coral pueden existir múltiples especies de Symbiodinium, una es dominante y otras están presentes con una densidad muy baja (Boulotte et al., 2016). Las diferentes especies de Symbiodinium que se han encontrado, poseen capacidades metabólicas y fisiológicas diferentes entre sí; por lo que su presencia dominante en el coral induce un fenotipo diferente en cuanto a productividad, beneficios metabólicos y resistencia al estrés ambiental. Se ha observado que durante el evento del blanqueamiento se pierde la microalga dominante $y$ en algunas ocasiones, las microalgas con abundancias bajas pueden repoblar los espacios (nichos) desocupados, suplir las funciones ecológicas y conferirle al coral la capacidad de sobrevivir a la condición de estrés que generó la supresión de la especie dominante (Hume et al., 2016). No obstante, cuando no se logra establecer una simbiosis nueva con las otras microalgas, el coral muere.

Recientemente, se ha propuesto que Symbiodinium establece también interacciones con bacterias simbiontes del coral. Se ha sugerido que esta triple interacción simbiótica (Coral-Symbiodinium-bacteria), confiere múltiples beneficios tanto a la microalga como al coral (Ainsworth et al., 2015; Bernasconi et al., 2019; Matthews et al., 2020; OcampoAlvarez et al., 2020). Por ejemplo, en estudios recientes de nuestro grupo de trabajo hemos observado que bacterias del género Salinispora, que son también simbiontes de coral, son capaces de establecer interacciones simbióticas no sólo con microalgas, sino que también con plantas superiores, donde le confieren a su nuevo huésped importantes beneficios fisiológicos, tales como promoción de crecimiento, foto-protección y protección al estrés oxidativo y a las altas concentraciones de salinidad (Ocampo-Alvarez et al., 2020). Por lo que es posible, que estas funciones de 
protección y crecimiento pudieran ser conferidas de igual manera al simbionte fotosintético Symbiodinium en el coral bajo condiciones de estrés relevantes.

Un tema poco explorado en los arrecifes de coral, son las implicaciones que tienen estos "terceros-jugadores", bacterias u hongos, en el balance, homeostasis y éxito ecológico de los corales. Además de esta triple interacción "coral-Symbiodinium-bacteria", es posible que existan múltiples interacciones simbióticas de dos, tres o más integrantes, tales como, coralbacteria, Symbiodinium-bacteria o bacteriabacteria, coral-hongo, entre otros, con beneficios biológicos y ecológicos, que en suma contribuyen a establecer en el coral fenotipos específicos de resiliencia que les permite resistir y persistir a determinadas condiciones ambientales, tales como el aumento de la temperatura del mar. Estas interacciones entre el coral y su microbioma le pueden brindar protección contra patógenos a través de una defensa química, resistencia al estrés ambiental a través de la activación de genes de respuesta al estrés e incremento de las capacidades metabólicas, como la capacidad de utilizar nutrientes de difícil disponibilidad (Peixoto et al., 2017). En este sentido, es entonces razonable pensar que las interacciones del coral con su microbioma le han conferido a estas especies, capacidades metabólicas y ventajas ecológicas competitivas para ser las especies dominantes y los bioconstructores principales de los ecosistemas de arrecifes durante millones de años (Bourne et al., 2016). Resulta intrigante pensar que durante ese largo periodo, donde han existido también fuertes variaciones climáticas, los corales y sus ecosistemas arrecifales no fueron eliminados y han persistido a través del tiempo. Sin embargo, en la actualidad, es bien conocido que los arrecifes de coral están sufriendo un gran impacto y que los corales quizá no serán capaces de soportar incrementos de tan solo $1^{\circ} \mathrm{C}$ por encima de su temperatura promedio del mar en verano (Hughes et al., 2018).

Los corales pueden modificar sus interacciones y con ello alcanzar un fenotipo nuevo que puede conferirle potencialmente la capacidad de adaptación a los cambios y sobrevivir. Pero entonces, ¿Por qué, los corales no logran sobrevivir a las condiciones de estrés térmico actuales?. Es posible, que algún otro factor que no estaba presente en el pasado, ahora contribuye a que los corales estén perdiendo su capacidad de resistir a cambios ambientales.

Un factor que coincide con el inicio de la degradación de los ecosistemas de arrecifes de coral y de muchos otros ecosistemas costeros de gran importancia ecológica como las praderas marinas, los bosques de macroalgas entre otros, es el uso extensivo de agroquímicos (Jones and Kerswell, 2003). El crecimiento de la población humana y la reducción y degradación de los suelos han hecho "necesario" incrementar artificialmente la eficiencia de los cultivos. La medida principal para lograr mejores rendimientos y calidad de los cultivos es la adición de productos agroquímicos, tales como, fertilizantes y herbicidas. Estos productos usados extensivamente, eventualmente son arrastrados al mar, donde alteran las características fisicoquímicas del agua y por ende, las relaciones ecológicas de los sistemas biológicos cercanos a la costa, como los ecosistemas de arrecife de coral.

El efecto de estos contaminantes es muy evidente, pues en estudios en los que se evalúan los microbiomas de coral de sitios que no tienen relación con descargas de efluentes con agroquímicos no presentan variación de su microbioma (Hernández-Zulueta et al., 2016). En contraste, los estudios que comparan los microbiomas de corales de sitios muy cercanos a las descargas de efluentes que contienen residuos de la agricultura, con los microbiomas de los corales de sitios alejados a las descargas, 
resultan tener diferentes ensamblajes microbianos (McDevitt-Irwin et al., 2017). Estas diferencias en los microbiomas deben ser ocasionadas por la presencia de herbicidas y fertilizantes disueltos en el agua de mar (Roitman et al., 2018).

Los herbicidas son utilizados en los sistemas de cultivo terrestre para eliminar plantas que compiten con las plantas de cultivo y suelen afectar diversas reacciones fotosintéticas, por lo que, pueden incrementar la vulnerabilidad de cualquier organismo fotosintético, incluyendo las microalgas endosimbiontes del coral (Negri et al., 2011; Wang et al., 2016).

Por otro lado, la presencia de fertilizantes agrícolas en la columna de agua de mar promueve el crecimiento de muchos organismos fotosintéticos, incluidas las microalgas marinas. Sin embargo, en estas condiciones, es de esperar un mayor crecimiento de especies con mayor capacidad de asimilación de estos nutrientes y con mayor tasa de crecimiento, con lo cual se establece un predominio sobre las especies de crecimiento lento, y se genera un desbalance entre las poblaciones existentes (Karcher et al., 2020). Por ejemplo, desplazando los organismos con la capacidad de establecer interacciones simbióticas con los corales, o bien, inhibiendo la cooperación entre los simbiontes potenciales con sus organismos huéspedes. Además, el exceso de biomasa de microalgas provocado por los fertilizantes eventualmente muere, lo que incrementa la cantidad de materia orgánica en la columna de agua y favorece el crecimiento de bacterias saprofíticas de rápido crecimiento que desplazan a las bacterias especializadas en rutas metabólicas complejas responsables de favorecer las simbiosis multitróficas en los corales. Así mismo, las bacterias saprofíticas al alimentarse de la materia orgánica consumen el oxígeno disuelto en la columna de agua, lo que provoca muerte por anoxia de muchos organismos marinos en la zona de afectación, incluidos los corales (Hughes et al., 2020;
Maher et al., 2019). De hecho, las zonas cercanas a sitios de descargas con concentraciones altas de fertilizantes en poco tiempo se convierten en zonas muertas (Hughes et al., 2020).

Si bien, el mar es inmenso y capaz de diluir una gran cantidad de compuestos, estos contaminantes se presentan como un gradiente que disminuye desde altas concentraciones cerca de la costa a bajas concentraciones mar adentro. Los arrecifes de coral y la mayoría de los ecosistemas marinos de mayor biodiversidad se encuentran cerca de las costas. Por lo tanto, es posible que los ensamblajes microbianos del pasado estén siendo modificados drásticamente debido a la contaminación marina por agroquímicos de las últimas décadas.

Si la contaminación marina por agroquímicos causa la eliminación de una gran proporción del stock de microorganismos especializados, microalgas y bacterias capaces de interactuar como simbiontes de los corales, entonces, la posibilidad que tiene el coral de seleccionar simbiontes nuevos y que éstos le confieran el fenotipo o las características necesarias para enfrentar una condición de estrés nueva, como las altas temperaturas, será cada vez menor.

Por otro lado, se sabe que los corales, así como las plantas, secretan compuestos orgánicos para atraer microorganismos que después pueden seleccionar para integrarlos como organismos simbiontes y que le permiten adaptarse a nuevas condiciones climáticas (Yamashita et al., 2014). Pero si ya existe alimento en exceso, como cuando hay contaminación por fertilizantes, estos microorganismos no necesariamente serán atraídos por los exudados del coral y la diversidad de los microorganismos se modifica al predominar microorganismos saprofíticos y de rápido crecimiento que no son capaces de interactuar o formar relaciones simbióticas con los corales (Zaneveld et al., 2016). 
Este escenario de degradación de los arrecifes de coral no es nada alentador, porque mientras continúe la contaminación por agroquímicos, el microbioma seguirá modificándose, los microorganismos capaces de interaccionar con los corales se seguirán eliminando del microbioma, por lo tanto, las poblaciones de corales continuarán disminuyendo debido a una aptitud biológica menor, conferida por los pocos microorganismos simbiontes de los que aún pueden disponer.

Este planteamiento desde luego no resta importancia al efecto negativo probado del cambio climático sobre los ecosistemas de coral. Por el contrario, se suma al efecto comprobado de la temperatura sobre la sobrevivencia de los corales. Por lo tanto, además de estudiar el mecanismo de acción de la temperatura sobre la sobrevivencia de los corales, también es necesario estudiar los mecanismos que influyen sobre las interacciones simbióticas del coral, las cuales contribuyen de igual manera a la disminución de su aptitud biológica ante el escenario del cambio climático. En consecuencia, el estudio de estas relaciones ecológicas contribuiría al diseño de estrategias integrales para disminuir y de ser posible, eliminar los episodios de mortalidad masiva de coral, así como para aumentar la capacidad de resiliencia de estos ecosistemas ante los inminentes efectos del cambio climático.

\section{Perspectiva y conclusiones}

Ante la situación negativa a la que están siendo sometidos los ecosistemas arrecifales que hemos puesto en perspectiva en este artículo, proponemos escenarios de investigación que aborden el tema desde un enfoque multi e interdisciplinario, que permita profundizar en el conocimiento detallado de los procesos y relaciones ecológicas dentro de un coral y su importancia en la resistencia y resiliencia de los ecosistemas de arrecife de coral.

Es menester que estas investigaciones tengan el alcance suficiente para descubrir los microorganismos capaces de establecer simbiosis verdaderas y generar posibles funciones relevantes para el coral. Las herramientas como metagenómica, metabolómica y bioinformática combinadas con análisis estadísticos robustos son relevantes para poder diferenciar la fracción transitoria de la fracción simbiótica permanente en el microbioma del coral. Además, las aproximaciones experimentales in vitro e in situ permiten comprobar si los microorganismos identificados potencialmente como simbiontes de corales, empleando técnicas moleculares y bioinformáticas, pueden establecer verdaderamente relaciones simbióticas y conferirles ventajas adaptativas a los corales.

Más aun, es necesario diseñar y ejecutar también experimentos a nivel de microcosmos, que involucren diferentes escenarios ambientales para entender el efecto y la respuesta del microbioma al interactuar con el coral. Por ejemplo, un estudio clave sería el aislamiento selectivo de los microorganismos potencialmente simbiontes (algas, bacterias) y la comprobación experimental del establecimiento de las simbiosis con corales axénicos, o con otros organismos superiores como las plantas. En estas interacciones inducidas, será necesario la evaluación de las propiedades emergentes de estas interacciones, orientadas a identificar beneficios sobre la aptitud biológica y ecológica de los huéspedes e incluso las potenciales aplicaciones biotecnológicas.

Lo anterior nos permitirá comprender mejor un proceso tan grave para el medio ambiente como el blanquimiento de coral, así como la capacidad intrínseca de resiliencia de los corales ante el estrés térmico. Esto con el fin de habilitar el diseño de estrategias para aliviar la 
degradación de los ecosistemas de coral. Por ejemplo, el uso de formulaciones probióticas diseñadas para ayudar a restituir la microbiota del coral con microorganismos adecuados para responder a un evento de estrés térmico (Rosado et al., 2019).

$\mathrm{O}$ alternativamente, reduciendo la presión por contaminación agroquímica en los ecosistemas arrecifales, para reestablecer el stock nativo de microorganismos simbiontes del coral. De este modo, el coral podría seleccionar de manera natural los microorganismos con los cuales puede establecer las interacciones simbióticas que les brinden mayor resistencia a los factores de estrés ambiental.

Quedan muchas preguntas aún por resolver, hipótesis que probar y un largo camino por recorrer para contribuir a la preservación de los ecosistemas de arrecifes de coral, pero lo podremos hacer más corto y lograr, si como investigadores, podemos establecer las interacciones multi e interdisciplinarias más aptas para afrontar este gran reto.

\section{Agradecimientos}

A CONACyT por su apoyo en el proyecto de Cátedras CONACyT 2196 otorgado a ABE, por el apoyo al proyecto de Ciencia Básica CB2015 - 257987 CONACyT otorgada a FARZ. También agradecemos a PRODEP por el apoyo al proyecto UDG-PTC-1460 otorgado a HOA; A la Universidad de Guadalajara por los apoyos PROSNI-2017 y P3E a HOA, Así como, PROSNI-2017-18 a ABE.

\section{Literatura citada}

Ainsworth, T.D., L. Krause, T. Bridge, G. Torda, J.B. Raina, M. Zakrzewski, ... y W. Leggat. (2015). The coral core microbiome identifies rare bacterial taxa as ubiquitous endosymbionts. ISME Journal, 9(10), 22612274. https://doi.org/10.1038/ismej.2015.39

Bernasconi, R., M. Stat, A. Koenders y M.J.
Huggett. (2019). Global Networks of Symbiodinium-Bacteria Within the Coral Holobiont. Microbial Ecology, 77(3), 794807. https://doi.org/10.1007/s00248-0181255-4

Boulotte, N.M., S.J. Dalton, A.G. Carroll, P.L. Harrison, H.M. Putnam, L.M. Peplow y M.J.H. Van Oppen. (2016). Exploring the Symbiodinium rare biosphere provides evidence for symbiont switching in reefbuilding corals. ISME Journal, 10(11), 26932701. https://doi.org/10.1038/ismej.2016.54

Bourne, D.G., K.M. Morrow y N.S. Webster. (2016). Insights into the Coral Microbiome: Underpinning the Health and Resilience of Reef Ecosystems. Annual Review of Microbiology, 70(1), 317-340. https://doi.org/10.1146/annurev-micro102215-095440

Davy, S.K., D. Allemand y V.M. Weis. (2012). Cell Biology of Cnidarian-Dinoflagellate Symbiosis. Microbiology and Molecular Biology Reviews, 76(2), 229-261. https://doi.org/10.1128/mmbr.05014-11

Fabina, N.S., H.M. Putnam, E.C. Franklin, M. Stat y R.D. Gates. (2013). Symbiotic specificity, association patterns, and function determine community responses to global changes: Defining critical research areas for coral-symbiodinium symbioses. Global Change Biology, 19(11), 3306-3316. https://doi.org/10.1111/gcb.12320

Hernández, A., F.A. Rodríguez-Zaragoza, M.C. García, J.M. Castro y Medina-Flores J. (2008). El manejo sostenible de los recursos pesqueros de la Reserva de la Biosfera Banco Chinchorro. WWF-México, $44 \mathrm{p}$.

Hernández-Zulueta, J., R. Araya, O. VargasPonce, L. Díaz-Pérez, A.P. RodríguezTroncoso, J. Ceh, ... y F.A: RodríguezZaragoza. (2016). First deep screening of bacterial assemblages associated with corals of the Tropical Eastern Pacific. FEMS Microbiol. Ecol. 92, fiw196. doi:10.1093/femsec/fiw196.

Hughes, D.J., R. Alderdice, C. Cooney, M. 
Kühl, M. Pernice, C.R. Voolstra y D.J. Suggett. (2020). Coral reef survival under accelerating ocean deoxygenation. Nature Climate Change, 10(4), 296-307. https://doi.org/10.1038/s41558-020-0737-9

Hughes, T.P., A.H. Baird, D.R. Bellwood, M. Card, S.R. Connolly, C. Folke y R. Grosberg. (2009). Climate change, human impacts, and the resilience of coral reefs. Science, 929(2003), 929-934. https://doi.org/10.1126/science.1085046

Hughes, T.P., K.D. Anderson, S.R. Connolly, S.F. Heron, J.T. Kerry, J.M. Lough, ... y S.K. Wilson. (2018). Spatial and temporal patterns of mass bleaching of corals in the Anthropocene. Science, 359(6371), 80-83. https://doi.org/10.1126/science.aan8048

Hume, B.C.C., C.R. Voolstra, C. Arif, C. D'Angelo, J.A. Burt, G. Eyal, ... y J. Wiedenmann. (2016). Ancestral genetic diversity associated with the rapid spread of stress-tolerant coral symbionts in response to holocene climate change. Proceedings of the National Academy of Sciences of the United States of America, 113(16), 4416-4421. https://doi.org/10.1073/pnas.1601910113

Jones, R.J. y A.P. Kerswell. (2003). Phototoxicity of Photosystem II (PSII) herbicides to coral. Marine Ecology Progress Series, 261, 149-159. https://doi.org/10.3354/meps261149

Karcher, D.B., F. Roth, S. Carvalho, Y.C. ElKhaled, A. Tilstra, B. Kürten, ... y C. Wild. (2020). Nitrogen eutrophication particularly promotes turf algae in coral reefs of the central Red Sea. PeerJ, 8:e8737. https://doi.org/10.7717/peerj.8737

Maher, R. L., M.M. Rice, R. McMinds, D.E. Burkepile, y R. Vega Thurber. (2019). Multiple stressors interact primarily through antagonism to drive changes in the coral microbiome. Scientific Reports 9, 1-12. doi:10.1038/s41598-019-43274-8.

Matthews, J.L., J.B. Raina, T. Kahlke, J.R. Seymour, M.J.H. van Oppen y D.J. Suggett. (2020).

Symbiodiniaceae-bacteria interactions: rethinking metabolite exchange in reef-building corals as multi-partner metabolic networks. Environmental Microbiology, 22(5), 1675-1687. https://doi.org/10.1111/1462-2920.14918

McDevitt-Irwin, J.M., J.K. Baum, M. Garren y R.L. Vega Thurber. (2017). Responses of coral-associated bacterial communities to local and global stressors. Frontiers in Marine Science, 4(Aug), 1-16. https://doi.org/10.3389/fmars.2017.00262

Negri, A.P., F. Flores, T. Röthig, y S. Uthicke. (2011). Herbicides increase the vulnerability of corals to rising sea surface temperature. Limnol. Oceanogr. 56, 471-485. doi:10.4319/lo.2011.56.2.0471.

Ocampo-Alvarez, H., I.D. Meza-Canales, C. Mateos-Salmón, E. Rios-Jara, F.A. Rodríguez-Zaragoza, C. Robles-Murguía, ... y A. Becerril-Espinosa. (2020). Diving Into Reef Ecosystems for Land-Agriculture Solutions: Coral Microbiota Can Alleviate Salt Stress During Germination and Photosynthesis in Terrestrial Plants. Frontiers in Plant Science, 11(May), 648. https://doi.org/10.3389/fpls.2020.00648

Peixoto, R.S., P.M. Rosado, D.C. de A. Leite, A.S. Rosado y D.G. Bourne. (2017). Beneficial microorganisms for corals (BMC): Proposed mechanisms for coral health and resilience. Frontiers in Microbiology, 8(Mar), 1-16. https://doi.org/10.3389/fmicb.2017.0034

Putnam, H.M., K.L. Barott, T.D. Ainsworth y R.D. Gates. (2017). The Vulnerability and Resilience of Reef-Building Corals. Current Biology, 27(11), R528-R540. https://doi.org/10.1016/j.cub.2017.04.047

Roitman, S., F. Joseph Pollock y M. Medina. (2018). Coral Microbiomes as Bioindicators of Reef Health. https://doi.org/10.1007/13836_2018_29

Rosado, P.M., D.C.A. Leite, G.A.S. Duarte, R.M. Chaloub, G. Jospin, U. Nunes da Rocha, ... y R.S. Peixoto. (2019). Marine probiotics: increasing coral resistance to 
bleaching through microbiome manipulation. ISME Journal, 13(4), 921-936. https://doi.org/10.1038/s41396-018-0323-6

Silveira, C. B., G. S. Cavalcanti, J. M. Walter, A. W. Silva-Lima, E. A. Dinsdale, D. G. Bourne, ... y F. L. Thompson. (2017). Microbial processes driving coral reef organic carbon flow. FEMS Microbiology Reviews, 41(4), 575-595. https://doi.org/10.1093/femsre/fux018

Stella, J. S., M.S. Pratchett, P.A. Hutchings y G.P. Jones. (2011). Diversity, importance and vulnerability of coral-associated invertebrates. Oceanography and Marine Biology: An Annual Review, 49, 43-116.

Spalding, M., L. Burke, S.A. Wood, J. Ashpole y J. Hutchison. (2017). Mapping the global value and distribution of coral reef tourism. Marine Policy, 82(May), 104-113. https://doi.org/10.1016/j.marpol.2017.05.01

Wang, C., X. Lin, L. Li y S. Lin. (2016). Differential growth responses of marine phytoplankton to herbicide glyphosate. PLoS ONE, 11(3), 1-20. https://doi.org/10.1371/journal.pone.015163

Yamashita, H., G. Suzuki, S. Kai, T. Hayashibara y K. Koike (2014). Establishment of coral-algal symbiosis requires attraction and selection. PLOS ONE, 9(5). e97003 https://doi.org/10.1371/journal.pone.0097003 Zaneveld, J.R., D.E. Burkepile, A.A. Shantz, C.E. Pritchard, R. McMinds, J.P. Payet, ... y R.V. Thurber. (2016). Overfishing and nutrient pollution interact with temperature to disrupt coral reefs down to microbial scales. Nature Communications, 7(May), 1-12. https://doi.org/10.1038/ncomms11833 